\title{
A Surgical Programme in Leprosy in Papua-New Guinea
}

\author{
J. K. A . GLEZY, F.R.G.s., F.R.A.c.s. \\ Department of Public Health, Madang, New Guinea
}

In February I 965 a surgical unit to deal with the deformities of leprosy was established in the modern General Hospital at Madang, on the nor th-east coast of New Guinea. Sixty beds and other appropriate accommodation was made available, and at the outset the expatriate staff consisted of the author, a nurse, and a physiotherapist, all of whom received training at Karigiri and Vellore in I 964. Although this unit has been functioning a comparatively short time, it is considered that a report of its development is justified by the social and geographic factors which had to be taken into account.

The Territory of Papua and New Guinea, comprising the eastern half of the island of New Guinea and the Bismarck and other archipelagoes, has a population of two million, half of whom live in the Highlands. With the exception of the sodden wastes of western Papua and the Sepik basin, virtually the whole of the Territory is rugged mountain country. Road systems are fragmentary at present, and for practical purposes all travel on the mainland, apart from patrolling, is done by air.

The estimated incidence of leprosy is $0.77 \%$, and of these one-fifth are lepromatous. The policy of this Department is to isolate infectious cases, and for this purpose there are about a dozen colonies spread over the country. These institutions have also served as homes for many non-infectious patients with various deformities.

It was realised years ago that a great deal could be done to prevent and ameliorate deformity in these patients, but the extension of leprosy services to include surgery was fraught with difficulty. Firstly, although some instances of persecution and murder have been reported, most leprosy patien ts suffer none of the ostracism which is so common in many other countries, and therefore they do not have the same cosmetic compulsion to co-operate in surgical and para-surgical care.
Secondly, the main centres of institutional leprosy work are far from general hospitals, and few of them offered anything like ideal conditions for surgical work.

Further, many of our patients are unwilling to leave their home ground, and it seemed that only the desperate and hopelessly crippled would be anxious to go to a distant centre for treatment. Even if patients were willing to travel, the high cost of air transport would make it essential that cases were accurately selected. While it is possible for any untrained person to send in a case of footdrop, selection of hand cases can only really be made by the surgeon, and it seemed that if adequate coverage of the Territory was to be achieved, the surgeon must travel, or risk waste of money and disappointment following transfer of unsuitable patients.

The great benefit of taking surgery to the patient has been amply demonstrated by Antia at Kondhwa and by Len nox at various places in South India, and it seemed desirable to develop a surgical service for leprosy in New Guinea on these lines.

The three prerequisites for such a service are good communications, good physiotherapy, and an acceptable minimum standard of operating and nursing facilities. The excellent commercial and mission air network eliminated the first problem, and the upgrading of facilities at a number of leprosaria with grants from The Leprosy Mission, and by various missions already staffing other leprosaria, has taken care of the third.

The second difficulty, the provision of good physiotherapy, was crucial, and more than one surgical service in leprosy has failed for want of it. We considered that adequate work could be done by nurses already working in leprosy, if they were trained in the essentials of the subject, with concentrated practice and supervision in the early stages. Accordingly a course was 
designed to teach the relevant anatomy (with cadaver demonstrations where possible), muscle assessment, the care of anaesthetic limbs, the prevention of deformities, the care of ulcers, and the basic physiotherapeutic techniques required for standard operations.

These courses, lasting three weeks, have been conducted in Madang, after which the trainees have gone home to prepare suitable cases for operation, and to apply their other new knowledge.

When all is ready the surgeon and nurse fly to the leprosarium and re-assess the cases with the local worker. We like to operate on about a dozen cases at each visit, and have found that nurses rapidly learn to select and prepare patients properly, so that we now are finding the first day's cases ready when we arrive.

At the conclusion of the visit a general inspection is made, prospective cases for the next session are examined, and old cases are reviewed. Particular problems are discussed with the local worker, and opportunities are taken to explain again and again to the assembled patients that trauma is responsible for ulceration and loss of tissue. We have found that this concept is readily grasped by most people, and the occasional rejection of a surgical candidate because of a blister or an apparently trivial wound emphasizes the importance we attach to limb care.

We have made a practice of re-inforcing the local worker's experience in Madang by sending our physiotherapist to ass ist with the first cases out of plaster, three weeks after operation. This visit lasts four or five days, and we have found that once is enough to give confidence and ensure reliability. We now have nurses in isolated places managing perfectly with such things as Brand's extensor-flexor many-tailed graft, tibialis posterior transfer, the sublimis operation, opponens replacement and other procedures.

This system has allowed the development of regular surgery at eight places away from Madang, only three of which are staffed by trained physiotherapis ts.

The benefits of this decentralisation are several. The general improvement in morale (of staff as well as patients) has been confirmed in every case. Frequent visitation enables us to see patients at all stages of their desease, and management of deformities, actual or incipient, can be better supervised than by rare visits. The essential health education principles concerning leprosy can be repeated to all, even though only a few may be operated on. Follow-up work becomes a possibility.

Another great advantage of decentralisation is that it allows many more personnel and patients to take part in the programme. At any one time we have i 50 or more patients receiving surgical treatment, which would be quite impossible in any single institution in this country. While the volume of work is a poor single criterion of any medical service's usefulness, we feel that the cost of such extensive travelling demands as high a return as is consistent with proper standards.

We frequently find that other surgical cases turn up during a visit to an outstation, and we are glad to exploit this situation to create a link between the leprosy service and the rest of the Department.

The most obvious hazard faced by an itinerant surgeon is the possibility that septic or other complications will arise in his absence, or for that matter in the absence of any doctor. In practice this has not been a problem so far, and it seems that the more isolated the hospital the rarer is the serious pathogen.

Clinical records have proved rather difficult to maintain when patients are widely scattered. A central set of notes is kept in Madang, and a copy of the relevant operation note and clinical photographs are sent to the peripheral institution to supplement the progress notes of the local worker. At each visit progress notes are made for addition to the Madang records, so that we may have a proper basis for follow-up of patients and procedures in the future.

It cannot be too strangly emphasised that surgery in leprosy stands or falls by the quality of the accompanying physiotherapy, and even then it forms but one facet of patient care. However because the results of operation are visible they command the patient's attention in a special way, and he is all the more ready to hear what we tell him about his disease. We have found that these people, many of whom are primitive by any standard, are most grateful for surg ical care, and are in fact coming to hospitals looking for correction of claw hands, even 
though they may never have bothered to come with a tuberculoid patch, the nature of which they know.

One of the special problems facing leprosy workers in New Guinea is the severe destruction of feet, seen particularly in the Highlands. While waiting for materials for the construction of the most suitable available pattern of shoe we have been using a variety of plastic sandal donated by The Leprosy Mission, and have found that these are acceptable when the patient understands that walking in bare feet will ruin his feet. Although this side of the work, being preventive in nature, is less spectacular than curative therapy where this is possible, we emphasize preventive care at every opportunity.

However, we belive that it is in the long run rather idle, and cold comfort, to tell a patient how to avoid injuring his limbs if we do nothing to correct muscle imbalance. Patients become weary of footdrop springs and hand exercises. We regard surgery as an essential part of any well-rounded leprosy programme, rather than a luxury. With energy and enthusiasm the cost of correcting a claw hand is at least comparable with repair of a hernia or a broken leg.

While we have relied heavily on mission nurses to do the physiotherapy in this programme, for teaching purposes and to deal withspecial problems in a large unit a widely experienced and fully equipped physiotherapist is essential. There will always be cases needing transfer to the central institution for more expert attention, but in practice we have found that these cases are remarkably few.

\section{S U M M A R Y}

The development of a $\mathrm{n}$ itinerant surgical service for leprosy patients in New Guinea is described. Experience has shown that the essential physiotherapeutic techniques can be taught to enthusiastic nurses in a short time, enabling them to maintain high standards of almost every aspect of prevention and care of the deformities seen in this country.

\section{A GKNOWLEDGEMENTS}

I am grateful to the World Health Organization for the grant of a fellowship which allowed me to spend 1964 in India, and to The Leprosy Mission for providing the nurse and physiotherapist and for the loan of a second physiotherapist for eight months in i965. Mr. W. R. McKeown (Secretary for Australia, The Leprosy Mission) first suggested this decentralised service, and Dr Paul Brand and Dr Grace Warren assessed our needs. Dr D. A. Russell, Senior Specialist Leprologist, Department of Public Health, T.P.N.G., has supported the programme from the beginning, and I am indebted to him for help at every stage.

I a m grateful to Dr R. F. R. Scragg, Director of Public Health, Territory of Papua and New Guinea, for permission to publish this report. 